\title{
EL DERECHO AL ACCESO A LA SEGURIDAD SOCIAL DE LOS TRABAJADORES AL SERVICIO DEL ESTADO*
}

\author{
THE RIGHT TO ACCESS TO SOCIAL SECURITY \\ OF THE STATE WORKERS
}
LE DROIT D'ACCES A LA SECURITE SOCIALE DES AGENTS DE L'ÉTAT

\author{
Karen Yarely GARCíA ARIZAGA* \\ Diana PÉREZ PADRÓN ${ }^{* * *}$
}

\begin{abstract}
RESUMEN: Los trabajadores al servicio del Estado representan un sector laboral desprotegido por diversas legislaciones burocráticas locales en materia de seguridad social; por mencionar un caso específico, los trabajadores temporales, pues son excluidos de la aplicación de la ley o no disfrutan de manera integral de ese derecho. El presente artículo tiene como principal objetivo demostrar el tratamiento discriminatorio que sufren los trabajadores burócratas en razón de la categoría asignada por la temporalidad de su nombramiento respecto de los trabajadores permanentes, pues en materia de seguridad social reciben una protección diferente, no obstante que ambos prestan un servicio personal y subordinado al Estado.
\end{abstract}

Se plantea como hipótesis que, para el reconocimiento del derecho humano a la seguridad social de los trabajadores burócratas, su categoría no debe ser un factor determinante, y que la legislación federal y local debe ser acorde al principio de igualdad, para garantizar el reconocimiento y acceso a este derecho. Para ello, utilizo el método comparativo para el análisis de las legislaciones locales tanto en materia burocrática como de

* Recibido el 21 de enero de 2019 y aprobado para su publicación el 25 de marzo de 2019.

** Profesora-investigadora en la Universidad Autónoma de Baja California.

*** Alumna del programa de maestría en ciencias jurídicas en la Universidad Autónoma de Baja California. 
seguridad social; asimismo, con el estudio del caso se describió la situación de vulnerabilidad de algunos sectores de estos trabajadores.

Palabras clave: trabajador, seguridad social, discriminación, protección, temporalidad.

ABSTRACT: Workers in the service of the State represent a labor sector unprotected by various local bureaucratic social security laws, to mention a specific case of temporary workers, since they are excluded from the application of the law or do not fully enjoy that right. The main objective of this article is to demonstrate the discriminatory treatment suffered by bureaucratic workers by reason of the category assigned by the temporary nature of their appointment with respect to permanent workers, given that in social security they receive different protection, despite the fact that both provide a personal service and subordinate to the State.

It is hypothesized that for the recognition of the human right to social security of bureaucratic workers their status should not be a determining factor and that federal and local legislation must be in accordance with the principle of equality to guarantee recognition and access to this straight. To do this, it used the comparative method for the analysis of local legislations in both bureaucratic and social security matters, also, the case study described the situation of vulnerability of some sectors of these workers.

Keywords: worker, social security, discrimination, protection, temporality.

RÉSUMÉ: Les travailleurs au service de l'État qui représente le secteur du travail non protégé par diverses lois bureaucratiques locales en matière de sécurité sociale, pour ne citer un cas particulier des travailleurs temporaires, ils sont exclus de l'application de la loi ou ne bénéficient pas de ce droit de manière holistique. Cet article a pour principal objectif de démontrer le traitement discriminatoire subi par les bureaucrates travailleurs parce que la catégorie attribuée par le caractère temporaire de sa nomination à l'égard des travailleurs permanents dans la sécurité sociale reçoivent une protection différente, mais ils ont tous deux rendent un personnel et subordonné au service de l'Etat.

On suppose que la reconnaissance du droit à la sécurité sociale des fonctionnaires de la classe ouvrière ne devrait pas être un facteur déterminant et que la législation fédérale et locale doit être conforme au principe de l'égalité pour assurer la reconnaissance et l'accès à cette à droite. Pour ce faire, il a utilisé la méthode comparative pour analyser les lois locales tant 
en matière de sécurité bureaucratiques et sociales aussi à l'étude de cas, la vulnérabilité de certains secteurs de ces travailleurs décrit.

Mots clés: travailleur, sécurité sociale, discrimination, protection, temporalité.

SUMARIO: I. Consideraciones preliminares y planteamiento del problema. II. El contenido de la seguridad social y el tratamiento de los trabajadores en las relaciones burocráticas en México. III. La seguridad social en el sistema jurídico mexicano e instrumentos internacionales. IV. Breve análisis comparativo entre entidades federativas. V. Los contratos regulados por la legislación civily los regímenes burocráticos especiales. VI. La incertidumbre de los trabajadores del sector salud. VII. Conclusiones y propuesta. VIII. Fuentes. IX. Anexos.

\section{CONSIDERACIONES PRELIMINARES Y PLANTEAMIENTO \\ DEL PROBLEMA}

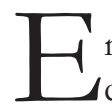
n el contexto internacional, la Constitución Política de los Estados Unidos Mexicanos de 1917 se ubica como la primera carta magna que reconoce derechos de carácter social, siendo el artículo 123 el referente por excelencia de los derechos de protección a la clase trabajadora a través del establecimiento de principios básicos que rigen las relaciones de trabajo.

Conforme a este precepto, toda persona tiene derecho al trabajo digno y socialmente útil; sin embargo, los principios a los que se hace referencia sólo beneficiaron en un primer momento a los trabajadores de la iniciativa privada, a fin de reconocer a su favor garantías sociales mínimas para alcanzar un nivel de vida digno: salario mínimo, jornadas máximas de ocho horas, salud, indemnizaciones por riesgos de trabajo, derechos colectivos, de seguridad social, entre otros, que se encuentran desarrollados en el apartado A del mencionado precepto, así como en su ley reglamentaria, la Ley Federal de Trabajo.

Por su parte, los trabajadores al servicio del Estado se mantuvieron por muchos años en la incertidumbre y desprotección social, sin derecho a disfrutar de garantías mínimas en su relación jurídica con la autoridad pública empleadora. Fue hasta 1960 cuando se incorporó al numeral 123 constitucional un segundo apartado, que estableció aquellas garantías, mismas que se encuentran desplegadas en su correspondiente ley reglamentaria: la Ley Federal de los Trabajadores al Servicio del Estado. 
Bajo esta óptica, derechos como la protección del salario, seguridad social y jornadas máximas de trabajo, encontraron un sustento formal, mientras que otros, como el de sindicación, huelga y la estabilidad en el empleo, se contemplaron de manera restringida, generando con esto un desequilibrio e incertidumbre en la relación jurídica entre éstos y las autoridades públicas empleadoras, puesto que no obstante la incorporación del apartado $\mathrm{B}$ y la expedición de la ley secundaria, a la presente fecha no puede hablarse de la mejora en la condición de empleado público.

El postulado anterior encuentra sustento al momento de analizar la facultad que poseen las entidades federativas para legislar en materia burocrática, pues los servidores públicos de las entidades federativas y municipios se rigen conforme a la Constitución local y de legislación secundaria correspondiente, en atención a lo dispuesto en los artículos 115, fracción VIII, párrafo segundo, y 116, fracción VI, de la Constitución federal, lo cual ha traído como consecuencia falta de congruencia entre Constituciones locales, al regular en forma diferenciada un derecho humano fundamental como lo es la seguridad social, reconociendo este derecho en algunos casos y en otros no, principalmente en atención a la categoría designada al trabajador (como lo son los trabajadores eventuales o supernumerarios), cuestión que encuentra relación en la estabilidad en el empleo.

En este sentido, se afirma que el hecho de que existan múltiples ordenamientos que regulen las condiciones laborales de los trabajadores al servicio del Estado no garantiza que, efectivamente, promuevan una mayor protección para estos, precisamente por el diferente tratamiento que reciben al establecer categorías entre los empleados públicos, distinguiendo y restringiendo, respecto de prerrogativas que constitucionalmente se encuentran previstas. Así pues, se plantea como principal cuestionamiento: ¿la categoría del trabajador burócrata debe considerarse para el acceso a los beneficios de seguridad social?, y a efecto de disipar esta interrogante se plantean como objetivos:

- Establecer conceptos fundamentales.

- Examinar normas de carácter internacional, así como legislación federal y local en materia de seguridad social y burocrática.

- Realizar una comparativa de las legislaciones burocráticas en las entidades federativas respecto de las diversas categorías de trabajadores, en razón a la temporalidad de la prestación de sus servicios y qué tipo de protección reciben. 
- Describir la transgresión del derecho a la seguridad social, así como del derecho de igualdad y no discriminación para los trabajadores al servicio del Estado; ello en relación con la temporalidad de la prestación de sus servicios para determinar si las leyes secundarias son inconstitucionales.

- Elaborar propuestas de solución al conflicto.

De este modo, se plantean como posibles hipótesis, que:

- La categoría del trabajador burócrata debe ser un factor independiente para el reconocimiento del derecho humano a la seguridad social.

- La congruencia entre legislación federal y local, respecto al derecho de igualdad y no discriminación en la regulación de las relaciones jurídicas entre los trabajadores burócratas y el Estado garantizará a los primeros el reconocimiento de su derecho a la seguridad social y el acceso a sus beneficios.

\section{EL CONTENIDO DE LA SEGURIDAD SOCIAL}

Y EL TRATAMIENTO DE LOS TRABAJADORES EN LAS RELACIONES BUROCRÁTICAS EN MÉXICO

Definir la seguridad social es una tarea compleja, pues no existe una noción uniforme que delimite exactamente los elementos, características y principios rectores a fin de establecer un modelo único. Para Fernando Ruiz Soberón, la seguridad social “...puede inscribirse en el concepto más amplio de Estado benefactor, entendido como las actividades del Estado en al menos cuatro grandes campos, como otorgar beneficios monetarios, cuidados de la salud, educación, alimentación, vivienda y otros servicios de bienestar". ${ }^{1}$

Por su parte, Gustavo Arce Cano señala que la seguridad social

...es el instrumento jurídico y económico que establece el Estado para abolir la necesidad y garantizar a todo ciudadano el derecho a un ingreso para vivir y a la salud, a través del reparto equitativo de la renta nacional y por medio de prestaciones del seguro social, al que contribuyen los patronos, los obreros y el Estado, o algunos de éstos, como subsidios, pensiones y atención facultativa, y de servicios sociales... ${ }^{2}$

1 Moreno Padilla, Javier, "Protección social y seguro social", en Kurczyn Villalobos, Patricia y Tena Suck, Rafael (coords.), Temas selectos de derecho laboral. Liber amicorum: Homenaje a Hugo Ítalo Morales Saldaña, México, UNAM, Instituto de Investigaciones Jurídicas, 2014, p. 369.

2 Arce Cano, Gustavo, De los seguros sociales a la seguridad social, México, Porrúa, 1972, p. 723. 
A su vez, organismos internacionales como la Organización Internacional del Trabajo (en adelante, OIT) la define como

la protección que la sociedad proporciona a las personas que la integran mediante una serie de medidas públicas, contra las privaciones económicas y sociales que, de no ser así, ocasionarían la pérdida o una fuerte reducción de los ingresos por causa de una enfermedad, accidente de trabajo, enfermedad laboral, desempleo, invalidez, vejez o muerte. ${ }^{3}$

De las anteriores precisiones teóricas se puede establecer que la seguridad social tiene un papel fundamental para combatir problemas como la pobreza, la inclusión social y, sobre todo, la desigualdad y brechas sociales, al prever una protección para todo individuo.

Como derecho de la persona, de carácter universal, la seguridad social se reconoce en los artículos 22 y 25 de la Declaración de los Derechos Humanos de la Organización Internacional de las Naciones Unidas (ONU), que la acoge como uno de los derechos humanos, en cuanto a "garantías jurídicas universales que protegen a los ciudadanos y a los grupos frente a las acciones que pueden menoscabar las libertades fundamentales y la dignidad humana" 4 y al ubicarse como un derecho humano, pero también social, presenta las características de un derecho inalienable, irrenunciable e inextinguible.

La seguridad social aparece en México en 1917 con la promulgación de la Constitución Política de los Estados Unidos Mexicanos, la llamada "Constitución social", por incorporar los primeros derechos sociales en los artículos 3, 27 y 123 por primera vez. En la fracción XXIX del artículo 123 apareció el primer esbozo de la seguridad social bajo la modalidad de cajas de seguros populares para los trabajadores del sector privado, que más adelante daría lugar a la expedición de una ley de seguro social de utilidad pública, que fue promulgada en 1943, bajo el nombre de Ley del Seguro Social, trayendo consigo la creación del organismo encargado de brindar la protección y servicios a toda la población: el Instituto Mexicano del Seguro Social (IMSS).

Por su parte, para los trabajadores al servicio del Estado existía la Dirección General de Pensiones Civiles y de Retiro, organismo público descentrali-

3 Moreno Padilla, Javier, "Protección social y seguro social", en Kurczyn Villalobos, Patricia y Tena Suck, Rafael (coords.), Temas selectos de..., cit., p. 372.

4 Declaración Universal de los Derechos Humanos, adoptada por la Asamblea General de las Naciones Unidas en París, Francia, el 10 de diciembre de 1948, disponible en: http:/ / wmm. un.org/es/universal-declaration-buman-rights/ (fecha de consulta: 24 de septiembre de 2018). 
zado con personalidad jurídica propia que surgió en 1925 (incluso, antes que el IMSS) con la expedición de la Ley General de Pensiones Civiles y Retiro, cuya finalidad era la administración de recursos de los trabajadores. Posteriormente, la Dirección se transformó en el Instituto de Seguridad y Servicios Sociales de los Trabajadores al Servicio del Estado a partir de la expedición de la ley de dicho instituto, el 28 de diciembre de 1959.5

Como se mencionó anteriormente, no fue hasta 1960 cuando se contempló la protección a los trabajadores al servicio del Estado a través de la incorporación del apartado B al artículo 123 constitucional, surgiendo las primeras bases normativas de la seguridad social para este tipo de empleados, no obstante las facultades de las entidades federativas y municipios para legislar en materia burocrática, lo que trajo como consecuencia una concepción diversa de la relación que tienen las autoridades públicas con sus empleados.

Así, tenemos, por un lado, diversas legislaciones que regulan las relaciones de los trabajadores al servicio de las entidades, de municipios, de organismos descentralizados, y por otro, leyes de seguridad social para este tipo de régimen laboral, sin dejar de lado los múltiples seguros sociales encargados de brindar este servicio público a los derechohabientes. La problemática radica en que cada legislación, en la que se reconocen de manera diferenciada los derechos de seguridad social, implica la diminución y, en ocasiones, el nulo acceso a este derecho humano.

Atendiendo a sus principios, la seguridad social es un derecho universal, pues la protección se brinda a toda persona sin importar la existencia de un vínculo laboral, y en el caso de los trabajadores, no debe estar sujeta a la calidad de trabajador ordinario o de trabajador burocrático, pues atendiendo a su fundamento constitucional, se encuentra previsto que ambos son protegidos, inclusive, a los que desempeñan labores de confianza. Según la doctrina, el principio rector de la seguridad social se entiende como el "... derecho que tiene toda persona por el hecho de ser humano a la seguridad social", ${ }^{6}$ el cual se apoya en principios operacionales y generales, siendo los primeros: la asistencia médica; el ingreso social de compensación; el ingreso que reemplaza el que se ha perdido y la inserción, reinstalación y valorización de los recursos

5 Guerrero Martínez, Daniel Enrique, La Ley del ISSSTE de marzo de 2007: el nuevo régimen de seguridad social de los trabajadores al servicio del Estado, México, Senado de la República e Instituto Tecnológico Autónomo de México, 2008, pp. 25-27.

6 Sánchez Castañeda, Alfredo y Morales Ramírez, María Ascensión, El derecho a la seguridad socialy a la protección social, México, UNAM, Instituto de Investigaciones Jurídicas, 2014, p. 17. 
humanos, ${ }^{7}$ lo que significa que como consecuencia de este derecho inherente a toda persona se contempla una protección que abarca los aspectos antes mencionados.

De este modo, abordaremos el derecho a la igualdad y no discriminación, que dentro de sus múltiples variantes se encuentra la no discriminación en el empleo. Este derecho trasciende, inclusive, a la forma de tener acceso a las prestaciones laborales; por ejemplo, las de seguridad social.

Lo que acontece para los trabajadores que desempeñen sus actividades de manera temporal, es que las prestaciones que recibe son limitadas, e incluso nulas, y ello atiende a la naturaleza de su nombramiento. Debe señalarse que en todas las legislaciones burocráticas se contemplan diversas modalidades de los nombramientos otorgados a los trabajadores; por mencionar algunos: por obra o tiempo determinado, interino, provisional, y aun cuando las funciones que realicen puedan ser de base o de confianza, impera la temporalidad del nombramiento, y por tanto, que dichos empleados queden excluidos de la aplicación de las leyes de seguridad social correspondientes.

En materia de derechos humanos, el artículo 1o. constitucional señala que se reconocen aquellos contenidos en la Constitución federal, así como los establecidos en tratados internacionales en los que México sea parte, y en forma paralela, se establecen obligaciones a cargo del Estado de respetar, promover, proteger y garantizar estos derechos a través de los ordenamientos secundarios, para que de esta forma se salvaguarden principios y derechos que dotan de orden y congruencia al sistema jurídico. Uno de estos derechos es el de seguridad social, que forma parte de los llamados derechos sociales.

Tenemos que la CPEUM, en el artículo 123, apartado B, señala que la seguridad social cubre los accidentes y enfermedades profesionales y no profesionales, maternidad, jubilación, invalidez, vejez y muerte; asimismo, la fracción XIV establece de manera expresa que las personas que desempeñen cargos de confianza disfrutarán de medidas protectoras del salario y de los beneficios de seguridad social. Mientras tanto, la Ley Federal de los Trabajadores al Servicio del Estado, en su artículo 3o., define al trabajador como toda persona que preste un servicio físico, intelectual o de ambos géneros, en virtud de un nombramiento expedido o por figurar en las listas de raya de los trabajadores temporales.

Es así que desde la Constitución federal se señala qué es lo que debe comprender este derecho, y que se hace extensivo a todos los trabajadores, tanto

\footnotetext{
7 Ibidem, p. 18.
} 
trabajadores de base y de confianza, y, por su parte, la Ley Federal de los Trabajadores al Servicio del Estado, en la definición anterior no excluye de su aplicación a los trabajadores por su temporalidad, sino que los contempla en la definición general de trabajador, y a contrario sensu, a nivel local es el tiempo de prestación de servicios lo que determina, de manera discriminatoria, si son sujetos de protección en las diversas leyes secundarias.

Atendiendo a la teoría garantista de Luigi Ferrajoli, existe un problema de eficacia en los derechos sociales al carecer de mecanismos que hagan efectivo su acceso:

...el problema de los derechos sociales es que no se han visto acompañados por garantías judiciales adecuadas... Lo que se ha hecho en materia de derechos sociales por los llamados Estados del bienestar no ha sido lo correcto, en tanto que han proliferado la burocracia, la discrecionalidad administrativa, el juego no regalado de los grupos de presión, el clientelismo, las sedes extralegales de solución de conflictos, la no transparencia del poder público; es decir, un Estado paternalista, burocrático, incapaz de ofrecer satisfacción a los derechos sociales. ${ }^{8}$

En este sentido, la seguridad social para los trabajadores al servicio del Estado presenta una cobertura limitada a aquellos que prestan servicios de manera temporal; es desigual en cada una de las entidades al brindar servicios y prestaciones diferentes a personas con riesgos análogos, lo que se debe, principalmente, a las condiciones laborales a las que se encuentran sujetos. Lo anterior se desprende de las legislaciones de Jalisco, Campeche, Oaxaca, Quintana Roo, entre otras, en las que se atenta contra el derecho de todo trabajador de ser sujeto de protección de la seguridad social, así, como el de igualdad y no discriminación, al considerar la temporalidad del nombramiento como una condición que conduce a su exclusión.

\section{LA SEGURIDAD SOCIAL EN EL SISTEMA JURÍDICO MEXICANO E INSTRUMENTOS INTERNACIONALES}

En México, la seguridad social encuentra su fundamento en el artículo 123, apartado A, fracción XXIX al establecer que la Ley del Seguro Social es

8 Ferrajoli, Luigi, en Cárdenas García, Jaime, La argumentación como derecho, México, UNAM, Instituto de Investigaciones Jurídicas, 2016, pp. 87 y 88. 
de utilidad pública y que comprende diversos seguros, como el de invalidez, vejez, vida, cesantía, enfermedades, accidentes, guarderías y cualquier otro "encaminado a la protección y bienestar de los trabajadores, campesinos, no asalariados y otros sectores sociales y sus familiares". 9 De la literalidad del texto se puede apreciar que, en principio, la seguridad social no se encuentra condicionada o dirigida a un sector determinado de trabajadores, mucho menos restringida, pues se señala para todos los trabajadores, inclusive se hace extensiva a sus familias.

Por otro lado, en las fracciones XI y XIV del apartado B se establecen las bases mínimas de la seguridad social para los trabajadores al servicio del Estado, mismas que de forma específica se detallan en la fracción XI, y que comprende la protección contra accidentes y enfermedades profesionales, no profesionales, maternidad, jubilación, invalidez, vejez, muerte, asistencia médica, medicinas, habitaciones, licencias, centros para vacaciones, de recuperación y tiendas económicas, tanto para los trabajadores como para sus familias. Mientras que la fracción XIV señala la garantía de seguridad social para los trabajadores de confianza.

Por su parte, la Ley del Instituto de Seguridad y Servicios Sociales de los Trabajadores del Estado, que es reglamentaria del artículo 123, apartado B, en su artículo 3o. establece que son de carácter obligatorio los seguros de salud (que comprende atención médica preventiva, atención médica curativa y de maternidad, y rehabilitación física y mental); de riesgos de trabajo; de retiro, cesantía en edad avanzada y vejez, y de invalidez y vida.

No obstante la normativa de carácter federal, los trabajadores de las entidades federativas, municipios, organismos descentralizados, entre otros, en materia de seguridad social, se encuentran sujetos a las disposiciones previstas en las leyes específicas en cada entidad, además de las que regulan regímenes burocráticos especiales, como fuerzas armadas, policías, entre otros; sin embargo, representa una obligación para el Estado la prestación de este servicio, por tratarse de un derecho social.

En el ámbito internacional, los derechos sociales son considerados cada vez con más fuerza por el conjunto de tratados y convenciones que trascienden, incluso, a su derecho interno. El Pacto Internacional de Derechos Económicos, Sociales y Culturales, en su artículo 9o., establece que los Es-

9 Constitución Política de los Estados Unidos Mexicanos, publicada en el Diario Oficial de la Federación el 5 de febrero de 1917, disponible en: http:/ / mmw.diputados.gob.mx/LeyesBiblio/ pdf/1_150917.pdf(fecha de consulta: 1o. de octubre de 2018. 
tados miembros tienen la obligación de garantizar a toda persona el derecho a la seguridad social a fin de proteger su dignidad humana, pues contribuye a la supervivencia de aquélla como de sus familias, al desarrollo personal, a la realización plena, a la integración social y cultural. ${ }^{10}$

El artículo 22 de la Declaración Universal de los Derechos Humanos establece que toda persona tiene derecho a la seguridad social y a obtener, mediante el esfuerzo nacional y la cooperación internacional, la satisfacción de los derechos económicos, sociales y culturales. ${ }^{11}$ Ello significa que en cada Estado deben adoptarse las medidas necesarias para que el servicio público de la seguridad a su cargo cumpla con las necesidades mínimas de la población, y, para el caso de ser insuficientes o implementar nuevos planes y programas, se debe contemplar la posibilidad de adoptar convenios, tratados o acuerdos regionales e internacionales para hacer efectivo este derecho. Por su parte, el artículo 25 de la citada Declaración se refiere al derecho de toda persona a los seguros en caso de desempleo, enfermedad, invalidez, viudez, vejez y otros casos que impliquen la pérdida de los medios de subsistencia.

El Convenio 102 de la Organización Internacional del Trabajo (OIT) sobre la seguridad social (norma mínima), insta a los Estados para que al momento de ratificar el Convenio garanticen por lo menos tres de las nueve ramas de prestaciones (asistencia médica de carácter preventivo o curativo; las prestaciones monetarias de enfermedad; por desempleo; de vejez; en caso de accidente de trabajo y enfermedad profesional; prestaciones familiares; de maternidad; invalidez, y de sobrevivientes), y, posteriormente, observar las obligaciones derivadas de otras ramas, a fin de cumplir con los propósitos del convenio y lograr el desarrollo progresivo de la protección que brinda a las personas. $^{12}$

El Protocolo Adicional a la Convención Americana sobre Derechos Humanos en materia de Derechos Económicos, Sociales y Culturales, conocido

10 Pacto Internacional de Derechos Económicos, Sociales y Culturales, adoptado por la Asamblea General de las Naciones Unidas el 16 de diciembre de 1966, disponible en: http:// www.ohchr.org/SP/ProfessionalInterest/Pages/CESCR.aspx (fecha de consulta: el 1o. de octubre de 2018.

11 Declaración Universal de los Derechos Humanos, proclamada por la Asamblea General de las Naciones Unidas en París el 10 de diciembre de 1948, disponible en: http:/ / wmw.un.org/ es/universal-declaration-buman-rights/ (fecha de consulta: 1o. de octubre de 2018).

12 Convenio sobre la Seguridad Social (norma mínima) núm. 102, adoptado por la Conferencia General de la Organización Internacional del Trabajo en Ginebra el 28 de junio de 1952, disponible en: http:// wmw.ilo.org/dyn/normlex/es/f?p=NORMLEXPUB:12100:0::NO: :P12100_INSTRUMENT_ID:312247 (fecha de consulta: 2 de octubre de 2018). 
como Pacto San Salvador, en su artículo 9o. contempla a la seguridad social como un derecho humano dentro del grupo de los llamados derechos sociales, mediante el cual una persona debe recibir protección frente a circunstancias que constituyan para ésta una limitación física o mental, como la desocupación, la vejez o la incapacidad, que impida la obtención de medios suficientes para lograr una vida digna. Asimismo, proporciona una segunda visión de este derecho, pues lo considera no sólo como aquel que es inherente a toda persona por el simple hecho de serlo, sino como consecuencia directa de contar con un trabajo, pues el segundo párrafo del artículo 9o. que en el caso de los trabajadores, la protección debe incluir por lo menos atención médica, un subsidio o jubilación, licencia de maternidad y el seguro de sobrevivencia para los descendientes de los trabajadores. ${ }^{13}$

De lo anteriormente señalado se puede observar que los diversos convenios, pactos y declaraciones reconocen como derecho fundamental, el derecho a la seguridad social, basado en la igualdad, en la dignidad humana, en la no discriminación, que debe imperar como característica de todo derecho reconocido; sin embargo, en la normativa interna del país vemos que las disposiciones regulan, en un sentido diverso, los alcances de este derecho, al grado de reconocer parcialmente en beneficio de un trabajador burócrata o excluirlo por completo de los regímenes de protección.

\section{BREVE ANÁLISIS COMPARATIVO ENTRE ENTIDADES FEDERATIVAS}

Para la realización del presente apartado se examinaron las legislaciones burocráticas y de seguridad social de las 32 entidades federativas, incluyendo la Ciudad de México, con objeto de identificar dos aspectos:

1. La clasificación de los trabajadores burócratas que se prevé en cada entidad (indicador 1).

2. La exclusión o excepción en la aplicación de las leyes de seguridad social que se establece para ciertas categorías de trabajadores (indicador 2).

13 Protocolo Adicional a la Convención Americana sobre Derechos Humanos en materia de Derechos Económicas, Sociales y Culturales, adoptado por la Asamblea General de la Organización de los Estados Americanos el 17 de noviembre de 1988, disponible en: http:/ / wnw.oas.org/juridico/spanish/tratados/a-52.html (fecha de consulta: 2 de octubre de 2018). 
Con relación al segundo punto, se analizó no sólo que los ordenamientos prevén un trato diferente a determinadas categorías de trabajadores, sino además se buscó detectar si de forma expresa se señala algún criterio que justifique dicha distinción. Un total de 71 leyes que regulan las relaciones laborales y las prestaciones de seguridad social de los trabajadores de cada entidad federativa, ayuntamientos, instituciones descentralizadas, organismos autónomos nos permitió estudiar diversas formas de clasificar a su personal, ya sea por el tipo de actividades que realizan, o por la duración en el servicio, la forma de contratación y de pago.

Sobre las categorías de trabajadores, se puede apreciar que existe diversidad en la denominación que reciben los trabajadores, haciendo distinciones, dependiendo del tipo de actividades que desempeñan (base o de confianza), de acuerdo con la temporalidad (eventuales, interinos, emergentes) y con relación a la legislación aplicable al contrato de que se trate (civil o mercantil).

Por lo que hace a la seguridad social, la legislación es explícita al momento de establecer quiénes son los sujetos de excepción a su aplicación; por un lado, de manera total al no considerarlos como trabajadores y sujetos de los beneficios que la ley otorga, y, por otro, de manera parcial, al señalar algunos casos en los que sólo serán sujetos de protección siempre que medie convenio para su afiliación a la institución de seguridad social de que se trate, o que sólo se verán beneficiados de algún tipo de seguro para cubrir determinados imprevistos.

Para facilitar el seguimiento del análisis ${ }^{14}$ se desarrollan los siguientes puntos.

\section{De los trabajadores de base y de confianza}

Como puede observarse, la mayoría de las legislaciones burocráticas en las entidades federativas contemplan ambas categorías, estableciéndose de manera genérica esta distinción en razón de la naturaleza de las actividades que ejecuten los trabajadores, y no por la simple denominación que se le dé al puesto. Para ambos se garantiza el derecho a la seguridad social, señalándose de manera particular que a los trabajadores de confianza les son respetadas las medidas de protección al salario y de seguridad social.

14 Véase tabla de resultados de los anexos. 
Esta revista forma parte del acervo de la Biblioteca Jurídica Virtual del Instituto de Investigaciones Jurídicas de la UNAM

\begin{tabular}{|c|c|}
\hline \multicolumn{2}{|r|}{$\begin{array}{c}\text { INDICADOR } 1 \\
\text { CLASIFICACIÓN DE TRABAJADORES BUROCRÁTICOS CONFORME } \\
\text { A LAS LEGISLACIONES BUROCRÁTICAS LOCALES }\end{array}$} \\
\hline Categoría & Entidades federativas \\
\hline De base & $\begin{array}{l}\text { Aguascalientes, Baja California Norte, Baja California Sur, } \\
\text { Campeche, Chiapas, Chihuahua, Coahuila, Colima, Durango, } \\
\text { Guanajuato, Guerrero, Hidalgo, Jalisco, Michoacán, Morelos, } \\
\text { Nayarit, Nuevo León, Oaxaca, Puebla, Querétaro, Quintana Ro, } \\
\text { San Luis Potosí, Sinaloa, Sonora, Tabasco, Tlaxcala, Veracruz, } \\
\text { Yucatán y Zacatecas. }\end{array}$ \\
\hline De confianza & $\begin{array}{l}\text { Aguascalientes, Baja California Norte, Baja California Sur, } \\
\text { Campeche, Chiapas, Chihuahua, Coahuila, Colima, Durango, } \\
\text { Estado de México, Guanajuato, Guerrero, Hidalgo, Jalisco, } \\
\text { Michoacán, Morelos, Nayarit, Nuevo León, Oaxaca, Puebla, } \\
\text { Querétaro, Quintana Roo, San Luis Potosí, Sinaloa, Sonora, } \\
\text { Tabasco, Tamaulipas, Tlaxcala, Veracruz, Yucatán y Zacatecas. }\end{array}$ \\
\hline
\end{tabular}

Un caso particular es el que se presenta en el estado de México, el cual contempla las categorías de "generales" y de "confianza", siendo la única entidad que omite la categoría de "base". También es de señalarse las variaciones que sufren las denominaciones; por ejemplo, en Coahuila y Tamaulipas existen los trabajadores "de base sindicalizados".

\section{INDICADOR 1}

CLASIFICACIÓN DE TRABAJADORES BUROCRÁTICOS CONFORME A LAS LEGISLACIONES BUROCRÁTICAS LOCALES

\begin{tabular}{|l|l|}
\hline Generales & Estado de México \\
\hline De base sindicalizados & Coahuila y Tamaulipas. \\
\hline
\end{tabular}

\section{Los trabajadores eventuales, supernumerarios, por obra,} y tiempos determinados y similares

Una práctica acostumbrada es la contratación que realiza el Estado para hacerse de trabajadores que ejecuten actividades de carácter eventual o especializadas, lo que implica para el Estado patrón evitar sostener una relación de trabajo permanente con aquéllos y crear categorías diversas para denominarlos. La siguiente tabla muestra cómo varían en cada entidad las formas de 
denominar a los trabajadores que prestan servicios por tiempos determinados, de manera transitoria o bajo la permanencia de la partida presupuestal que corresponda.

\section{INDICADOR 1}

CLASIFICACIÓN DE TRABAJADORES BUROCRÁTICOS CONFORME

A LAS LEGISLACIONES BUROCRÁTICAS LOCALES

\begin{tabular}{|l|l|}
\hline Supernumerarios & $\begin{array}{l}\text { Baja California Sur, Campeche, Colima, Durango, Quintana } \\
\text { Roo, Sinaloa y Tamaulipas. }\end{array}$ \\
\hline Temporales & $\begin{array}{l}\text { Aguascalientes, Guanajuato, Guerrero, Jalisco, Michoacán, } \\
\text { Puebla y Zacatecas. }\end{array}$ \\
\hline Accidentales & Aguascalientes. \\
\hline Interinos & Chiapas, Guanajuato y Tlaxcala. \\
\hline Eventuales & Chiapas, Chihuahua, Morelos, Querétaro y San Luis Potosí. \\
\hline Extraordinarios & Chihuahua y Tamaulipas. \\
\hline $\begin{array}{l}\text { De obra y tiempo } \\
\text { determinado }\end{array}$ & Tabasco y Tlaxcala. \\
\hline
\end{tabular}

En el caso de Jalisco, los trabajadores se clasifican bajo dos criterios: por la naturaleza de la función y por la temporalidad del nombramiento, entre los que se encuentran los definitivos, temporales (generalmente conocidos como supernumerarios), que a su vez se dividen en interinos, provisionales, por tiempo y obra determinada ${ }^{15}$ por lo que el solo hecho de tener dicho carácter, automáticamente los coloca al margen de la protección de la Ley del Instituto de Pensiones del Estado de Jalisco.

La contratación por tiempo u obra determinada, de conformidad con diversas legislaciones, atiende a varios supuestos: cuando lo exige la naturaleza del trabajo; la sustitución de un trabajador de forma temporal (como los interinos), y en el caso de incremento de trabajo por temporadas. Sin embargo, la contratación de este tipo de personal en la práctica no se ajusta a los criterios señalados, a pesar de ser una figura muy utilizada, pues en ocasiones la materia o necesidad del servicio subsiste y se transforma en servicios transitorios.

Con relación a la aplicación de las normas de seguridad social, el factor de temporalidad de la relación de trabajo impacta en gran medida para el disfrute

15 Ley para los Servidores Públicos del Estado de Jalisco, artículo 3o., disponible en: https:// wmm.jalisco.gob.mx/sites/default/files/ley-servidores-jalisco.pdf (fecha de consulta: 1o. de diciembre de 2018). 
de los beneficios de este servicio público, así como en el derecho humano mismo. De la siguiente descripción de las legislaciones se advierte que se alejan de los principios que rigen para la observancia de este derecho, pues se excluye de los beneficios a los trabajadores temporales, eventuales, transitorios y similares, dejando en completa desprotección a un número considerable de servidores públicos, no obstante de la existencia de una relación de trabajo con una subordinación, por lo que deben ser sujetos de aseguramiento sin importar el tiempo de la relación que se trate, pues son de igual forma propensos a los riegos que todo servidor está expuesto durante la prestación del servicio.

\begin{tabular}{|l|l|}
\hline \multicolumn{2}{|c|}{$\begin{array}{c}\text { INDICADOR 2 } \\
\text { EXCLUSIÓN-EXCEPCIÓN DE LA APLICACIÓN DE LA LEGISLACIÓN } \\
\text { BUROCACA LOCAL EN MATERIA DE SEGURIDAD SOCIAL }\end{array}$} \\
\hline \multicolumn{1}{|c|}{ Entidad federativa } & \multicolumn{1}{|c|}{ Exclusión o excepción de aplicación de la normatividad } \\
\hline Campeche & $\begin{array}{l}\text { Los contratados bajo la legislación común o sujetos a } \\
\text { honorarios, trabajadores eventuales y de lista de raya. }\end{array}$ \\
\hline Coahuila & $\begin{array}{l}\text { Los que se encuentran sujetos a honorarios, por contrato } \\
\text { de obra, contrato civil, interinatos, trabajadores de la } \\
\text { educación, los que prestan servicios eventuales. }\end{array}$ \\
\hline Jalisco & $\begin{array}{l}\text { Los que prestan servicios mediante contrato por tiempo y } \\
\text { obra determinados y por contrato civil. }\end{array}$ \\
\hline Michoacán & $\begin{array}{l}\text { Los que prestan servicios por obra o a plazo fijo, a lista de } \\
\text { raya, eventuales o emergentes y los sujetos a honorarios. }\end{array}$ \\
\hline Nayarit & $\begin{array}{l}\text { Los que prestan servicios por tiempo y obra determinados } \\
\text { y los sujetos a la legislación común. }\end{array}$ \\
\hline Nuevo León & $\begin{array}{l}\text { Los que prestan servicios por honorarios o contrato } \\
\text { civil, eventuales con vigencia menor a seis meses y los } \\
\text { que prestan servicios por un tiempo menor a diez horas } \\
\text { semana-mes. }\end{array}$ \\
\hline Oaxaca & $\begin{array}{l}\text { Los que prestan servicios eventuales, los que cotizan en } \\
\text { un régimen distinto al previsto en la ley y los que perciban } \\
\text { honorarios. }\end{array}$ \\
\hline Puebla & $\begin{array}{l}\text { Los que prestan servicios por honorarios, contrato } \\
\text { de legislación común (excepto que medie convenio), } \\
\text { contratos eventuales menores a seis meses. Aquellos que } \\
\text { ingresen al servicio por primera vez y hayan cumplido } \\
\text { cincuenta años sólo tienen derecho al servicio médico. }\end{array}$ \\
\hline
\end{tabular}


Esta revista forma parte del acervo de la Biblioteca Jurídica Virtual del Instituto de Investigaciones Jurídicas de la UNAM http://www.juridicas.unam.mx/

\begin{tabular}{|l|l|}
\hline \multicolumn{1}{|c|}{ Entidad federativa } & \multicolumn{1}{|c|}{ Exclusión o excepción de aplicación de la normatividad } \\
\hline Quintana Roo & $\begin{array}{l}\text { Los que prestan servicios por honorario, contrato civil y } \\
\text { trabajadores eventuales. }\end{array}$ \\
\hline Tabasco & $\begin{array}{l}\text { Los prestadores de servicios profesionales, contratados } \\
\text { por la legislación civil y trabajadores eventuales (salvo } \\
\text { convenio). }\end{array}$ \\
\hline Yucatán & $\begin{array}{l}\text { Los que prestan servicios por contrato civil o laboral, } \\
\text { trabajadores a lista de raya y los que prestan servicios } \\
\text { eventuales o emergentes. }\end{array}$ \\
\hline
\end{tabular}

De la tabla anterior se observa que las legislaciones de forma expresa excluyen a un gran número de categorías de trabajadores en razón de nombramientos asignados de forma temporal, lo que crea, por un lado, la incertidumbre respecto de su estabilidad en el empleo, pues el trabajador desconoce si será contratado nuevamente, y, por otro lado, se ve afectado, porque no le resultan aplicables las disposiciones de seguridad social, o sólo de forma parcial, como es el caso de Nuevo León, donde los trabajadores eventuales con un tiempo menor de seis meses sólo tienen derecho al seguro de enfermedades y de maternidad, ${ }^{16}$ o sólo el seguro médico, mediante convenio en Puebla ${ }^{17}$ y Tabasco. ${ }^{18}$

Del mismo modo, los trabajadores en lista de raya se ven excluidos en ciertas legislaciones. Como es de recordar, la lista de raya controla a los trabajadores temporales por obra o tiempo determinado, lo que significa que aquel que desarrolle actividades en dicha modalidad no será acreedor de las prestaciones y servicios de seguridad social. Otros factores, claramente discriminantes, es que las propias leyes establecen que no consideran como servidores públicos o trabajadores a las personas trabajadoras de manera eventual.

16 Ley del Instituto de Seguridad y Servicios Sociales de los Trabajadores del Estado de Nuevo León, artículo 4o., disponible en: http://mmm.hcnl.gob.mx/trabajo_legislativo/leyes/pdf/ LEY\%20DEL\%20SERVTCIO\%20CIVIL\%20DEL\%20ESTADO\%20DE\%20NUEVO\%20 LEON.pdf (fecha de consulta: 1o. de diciembre de 2018).

17 Ley del Instituto de Seguridad y Servicios Sociales de los Trabajadores al Servicio de los Poderes del Estado de Puebla, artículo 4o., disponible en: http:// wmw.ordenjuridico.gob.mx/ Documentos/Estatal/Puebla/wo96681.pdf (fecha de consulta: 1o. de diciembre de 2018).

18 Ley de Seguridad Social del Estado de Tabasco, artículo 2, disponible en: bttps:// transparencia.tabasco.gob.mx/media/CGDRPE/2015/4/295049.pdf (fecha de consulta: 1o. de diciembre de 2018). 
Sin importar la categoría que el trabajador desempeñe (de base o de confianza), mientras se expidan nombramientos temporales o se asimilen relaciones laborales a una mera prestación de servicios profesionales, como se ha podido analizar en las legislaciones anteriores, a los trabajadores se les vulnera total o parcialmente el derecho a la seguridad social.

El problema no sólo estriba en la exclusión de estos servidores públicos, sino que atendiendo a los textos normativos no se contempla la obligatoriedad de su inscripción, por lo que al prolongarse ésta se deja en incertidumbre al servidor público sobre los derechos que trae aparejados el hecho de cotizar en un seguro social, como lo son la pérdida de la oportunidad de generar semanas cotizadas, y que a la postre genere el derecho a una pensión, así como el gozar de prestaciones económicas y en especie que garanticen un nivel de vida digno para el servidor público y su núcleo familiar ante una contingencia, como lo puede ser un riesgo de trabajo, una enfermedad profesional o general, la maternidad, la invalidez o vejez, la cesantía, y en ocasiones la muerte.

\section{LOS CONTRATOS REGULADOS POR LA LEGISLACIÓN CIVIL Y LOS REGÍMENES BUROCRÁTICOS ESPECIALES}

A manera de referencia, se hace alusión a este tipo de trabajadores, que en virtud de la relación contractual o por disposición de ley se encuentran como caso de excepción a la legislación de seguridad social en el ámbito burocrático. Por regla general, el nombramiento es la forma más usual de nacimiento de la relación laboral entre el Estado y sus trabajadores para la prestación de los servicios necesarios, para dar cumplimiento con sus funciones.

Como excepción se encuentra la contratación de profesionistas, con los que se establece una relación de naturaleza civil o mercantil, según el caso. Sin embargo, puede acontecer que el Estado utilice este tipo de contratación para evitar dar cumplimiento a sus obligaciones como patrón, por lo que decide crear relaciones de tipo administrativo o civil bajo el régimen de honorarios, asimilados al salario, y de esta manera no inscribir a su personal a los institutos encargados de la seguridad social.

Respecto a los regímenes burocráticos especiales, la normatividad señala que éstos se verán regulados por su propia legislación, como los trabajadores de la educación y de instituciones policiales, por lo que sus regímenes de seguridad social también serán determinados por dicha legislación. 


\section{LA INCERTIDUMBRE DE LOS TRABAJADORES}

DEL SECTOR SALUD

Un grupo de trabajadores que se ha visto afectado es el personal eventual que cubre incidencias de las secretarías de Salud en las entidades federativas. Algunas de estas personas contaban con más de quince años laborando bajo esta categoría sin reconocerles el derecho de obtener una plaza o base dentro de la dependencia, lo que los colocaba en una situación de incertidumbre ante la falta de prestaciones laborales, así como prestaciones en materia de seguridad social.

Las condiciones en las que laboraban tanto médicos, enfermeros, administrativos y supernumerarios de la Secretaria de Salud de Jalisco eran precarias. A los trabajadores de hospitales, como Valentín Gómez Farías (dependiente del Instituto de Seguridad y Servicios Sociales), se les negaba la atención médica y, a otros, que laboraban en el Hospital General de Occidente, no recibían su salario en las condiciones pactadas, ${ }^{19}$ por lo que la necesidad de que el gobierno asegurara a sus empleados los derechos que les corresponde como cualquier otro trabajador se convirtió en una exigencia social.

Como consecuencia, los trabajadores decidieron agruparse y formar un sindicato, realizar protestas y dar a conocer las condiciones en las que desarrollan sus actividades bajo esquemas de contratación que vulneran derechos laborales. Israel González González, uno de los líderes del Sindicato Independiente del Sector Salud, por sus siglas SISS, manifestó que es común que las autoridades del sector salud contraten en demasía a personal supernumerario para evitar responsabilidades y obtener el mismo trabajo a cambio de pagar menos; además, señaló: "Ya se acostumbraron a que el trabajador supernumerario les haga el trabajo sin tener derecho a ninguna prestación, con pagos incompletos". ${ }^{20}$

El pasado 17 de mayo de 2017, el gobierno de Jalisco anunció la creación de 101 plazas de base a una parte del personal cubreincidencias, que se otorgarían bajo los criterios establecidos en la convocatoria respectiva, entre los cuales destaca la categoría, antigüedad y acreditación del nivel profesional del aspirante. En palabras del líder de la sección 28 del Sindicato Nacional de

19 "Empleados de segunda en el sector salud", Proceso, 11 de enero de 2014, disponible en: https:/ / wmm.proceso.com.mx / 362154/empleados-de-segunda-en-el-sector-salud (fecha de consulta: 22 de noviembre de 2018).

20 Idem. 
Trabajadores del Sector Salud, Jesús Lépez Ponce “...a partir del momento que se les dé el nombramiento con su nuevo estatus, ellos dejarán de ser trabajadores de contrato eventual y tendrán todas las prestaciones de una plaza de base". ${ }^{21}$ Sin duda, esto representa un logro para este grupo de trabajadores, con el propósito de regular su situación laboral; significa crear certidumbre para el trabajador burócrata.

Esto sólo es una parte de la realidad en la que se desenvuelven los trabajadores sujetos a regímenes y categorías que limitan o restringen por completo su derecho humano a la seguridad social, pues resulta más preocupante el hecho de que se traduce en la afectación a uno de los servicios más básicos para la sociedad como lo es el de salud, lo que a futuro desmotiva al trabajador en la prestación de sus servicios, al verse vulnerado en sus derechos más mínimos.

¿Qué sucede en otras entidades? En Zacatecas, desde 2013, las protestas de trabajadores eventuales de los servicios de salud aumentaban, pues venían laborando por más de seis años sin recibir seguridad social y percibían ingresos muy bajos. Enfermeras, médicos y administrativos señalaron que se les recomendaba afiliarse al Seguro Popular "pero todo mundo sabe que no sirve", "más de 50 por ciento de las enfermeras y los médicos somos eventuales...", 22 comentaban.

Para 2017, un tema que se volvió parte importante de la agenda pública del gobierno zacatecano fue otorgar seguridad social para los trabajadores del Seguro Popular, y que no sólo afectaba a esa entidad, sino a las demás, por lo que era necesario un análisis de todas las legislaciones en la materia. ${ }^{23}$

\section{CONCLUSIONES Y PROPUESTA}

A manera de conclusión, del análisis realizado se advierte que las legislaciones antes expuestas son contrarias a lo previsto por la Constitución federal

21 Secretaría de Salud del Estado de Jalisco, "Anuncia SSJ 101 plazas de base para cubre incidencias", 17 de mayo de 2017, disponible en: https://ssj.jalisco.gob.mx/prensa/noticia/6916 (fecha de consulta: 22 de noviembre de 2018).

22 "Trabajadores del sector salud de Zacatecas, sin seguridad social ni estabilidad laboral", La Jornada, 10 de septiembre de 2013, https:/ / mmw.jornada.com.mx/2013/09/10/ estados/032n1est, consultado el 25 de noviembre de 2018.

23 La Jornada Zacatecas, sitio web, nota periodística "Tema prioritario, dar seguridad social a trabajadores de Seguro Popular: López”, del 24 de octubre de 2017, disponible en: bttp:/ ljz: $m x / 2017 / 10 / 24$ / tema-prioritario-dar-seguridad-social-a-trabajadores-de-seguro-popular-lopez/ (fecha de consulta: 25 de noviembre de 2018). 
y tratados internacionales en materia de seguridad social, pues los servidores públicos que prestan sus servicios con carácter de eventual, temporales, supernumerarios, por obra o tiempo determinado, no tienen acceso a las prestaciones de seguridad social que se les brinda a aquellos de base o de confianza, lo que desde luego es discriminatorio, pues se toma como referente para hacer dicha distinción la temporalidad del nombramiento que se les otorga, lo que lleva a confirmar la hipótesis planteada.

Toda persona tiene derecho a un trabajo digno, el cual comprende, entre otros aspectos, el acceso a la seguridad social. Aun cuando los trabajadores al servicio del Estado desempeñan una obligación mediante cargo o comisión, a su vez son sujetos de derechos laborales y de seguridad social. Dichas prestaciones son irrenunciables, y en términos del artículo 123, apartado B, es obligación de las entidades o dependencias, asegurar el acceso a la seguridad social, con independencia del tipo nombramiento que se les otorgue.

Los principales argumentos que lleva a justificar la distinción y exclusión a ciertos servidores públicos respecto del acceso a la seguridad social radican en la calidad que tiene el servidor, y, por otro lado, el discurso políticofinanciero, en el sentido de la inviabilidad de otorgar prestaciones a todo el personal de cada dependencia; sin embargo, las anteriores manifestaciones carecen de sustentabilidad.

Lo anterior se afirma porque ha quedado establecido en múltiples convenios internacionales que la seguridad social, como derecho humano, debe ser garantizado a toda persona por el simple hecho de serlo, por lo que no puede incidir en su reconocimiento el hecho de tener la calidad de trabajador público o privado, pues es un derecho reconocido a nivel constitucional, que implica el acceso a una serie de prestaciones mínimas que puede ser extensivas, pero no disminuidas, en leyes secundarias; ello, en atención al principio de progresividad de los derechos humanos. En el mismo sentido, vista la seguridad social como un derecho de carácter prestacional, es un servicio público a cargo del Estado, por lo que éste es el primer responsable y único garante de aquél, y aunado a la calidad de patrón respecto a los servidores públicos debe garantizarlo y costearlo, por lo que si no puede hacer frente a esta exigencia social a través de los seguros sociales burocráticos, habrá que considerar la prestación de este servicio por conducto del IMSS y, en su caso, de instituciones de carácter privado.

En este sentido, las dependencias deben realizar las modificaciones necesarias para llevar a cabo la inscripción de los trabajadores temporales, even- 
tuales, supernumerarios o bajo el nombre asignado, ante la institución de seguridad social correspondiente y realizar las aportaciones respectivas en su carácter de patrón. De esta manera, el aspecto económico se verá reforzado, pues serán las aportaciones tanto del Estado patrón como de sus trabajadores las que se vean reflejadas en los ingresos de los organismos de seguridad social.

Como propuesta de solución a esta problemática se plantea el análisis de una reforma en cada uno de los cuerpos normativos que contemplan esta distinción, a fin de eliminar esta práctica legislativa, que deja en desamparo a dichos servidores. El ejemplo más sencillo es armonizar las legislaciones locales con su análoga a nivel federal, la Ley del Instituto de Seguridad y Servicios Sociales de los Trabajadores al Servicio del Estado, que en su artículo 6o., fracción XXIX, establece quiénes tienen el carácter de trabajador para efectos de la aplicación de la ley, y de forma expresa señala que son los que "presten sus servicios en las Dependencias o Entidades, mediante designación legal o nombramiento, o por estar incluidas en las listas de raya de los trabajadores temporales", ${ }^{24}$ y que incluso se hace extensiva al personal contratado bajo la legislación civil. De esta manera, al armonizar la normatividad secundaria se evitaría dejar en desprotección al personal que por su condición de trabajador temporal se ha visto excluido en los términos que se han discutido previamente.

La condición para tener una regulación de las condiciones laborales en el empleo público es la observancia de principios y derechos bajo la perspectiva de no discriminación, lo que implica que para todos los trabajadores se debe reconocer el derecho a la seguridad social, con independencia de las actividades que desarrollen y la temporalidad de su encargo; ello, para que exista armonía y congruencia con la legislación federal y con los tratados internacionales, aclarando que lo antes propuesto no implica, necesariamente, la pérdida de la autonomía de las entidades federativas con relación a su facultad de legislar en materia burocrática. Recordemos que la seguridad social es un derecho social a cargo del Estado y exigible a éste, por lo que es necesario crear mecanismos para su acceso.

24 Ley del Instituto de Seguridad y Servicios Sociales para los Trabajadores del Estado, publicada en el Diario Oficial de la Federación, el 31 de marzo de 2007, disponible en: http:/ / www.diputados.gob.mx/LeyesBiblio/pdf/LISSSTE_220618.pdf(fecha de consulta: 20 de octubre de 2018). 


\section{FUENTES}

ArCE CANO, Gustavo, De los seguros sociales a la seguridad social, México, Porrúa, 1972.

CÁRDENAS GARCíA, Jaime, La argumentación como derecho, México, UNAM, Instituto de Investigaciones Jurídicas, 2016.

GUERRERO MARTÍNEZ, Daniel Enrique, La Ley del ISSSTE de marzo de 2007: el nuevo régimen de seguridad social de los trabajadores al servicio del Estado, México, Senado de la República-Instituto Tecnológico Autónomo de México, 2008.

Kurczyn Villalobos, Patricia y TenA SuCK, Rafael (coords.), Temas selectos de derecho laboral. Liber amicorum: homenaje a Hugo Ítalo Morales Saldaña, México, UNAM, Instituto de Investigaciones Jurídicas, 2014.

RuIz Moreno, Ángel Guillermo, El nuevo derecho de la seguridad social, México, Porrúa, 2017.

SÁnCHEZ CASTAÑEDA, Alfredo y MORALES RamíreZ, María Ascensión, El derecho a la seguridad social y a la protección social, México, UNAM-Porrúa, 2014.

\section{Informáticas}

La Jornada, "Trabajadores del sector salud de Zacatecas, sin seguridad social ni estabilidad laboral", del 10 de septiembre de 2013, disponible en: https:/ / www.jornada.com.mx/2013/09/10/estados/032n1est.

La Jornada Zacatecas, "Tema prioritario, dar seguridad social a trabajadores de Seguro Popular: López”, del 24 de octubre de 2017, disponible en: bttp:/ / lj:mx/2017/10/24/tema-prioritario-dar-seguridad-social-a-trabajadoresde-seguro-popular-lopez/.

Proceso, "Empleados de segunda en el sector salud", del 11 de enero de 2014, disponible en: https://www.proceso.com.mx/362154/empleados-de-segunda-enel-sector-salud.

SECRETARÍA DE SALUD DEL ESTADO DE JALISCO, "Anuncia SSJ 101 plazas de base para cubre incidencias", del 17 de mayo de 2017, disponible en: https://ssj.jalisco.gob.mx/prensa/noticia/6916. 


\section{ANEXOS}

TABLA 1

ELABORACIÓN PROPIA CON DATOS DE LA LEGISLACIÓN BUROCRÁTICA LOCAL Y EN MATERIA DE SEGURIDAD SOCIAL EN LAS ENTIDADES FEDERATIVAS

\begin{tabular}{|c|c|}
\hline \multicolumn{2}{|c|}{$\begin{array}{c}\text { INDICADOR } 1 \\
\text { CLASIFICACIÓN DE TRABAJADORES BUROCRÁTICOS CONFORME } \\
\text { LAS LEGISLACIONES BUROCRÁTICAS LOCALES }\end{array}$} \\
\hline Categoría & Entidades federativas \\
\hline De base & $\begin{array}{l}\text { Aguascalientes, Baja California Norte, Baja California } \\
\text { Sur, Campeche, Chiapas, Chihuahua, Coahuila, Colima, } \\
\text { Durango, Guanajuato, Guerrero, Hidalgo, Jalisco, } \\
\text { Michoacán, Morelos, Nayarit, Nuevo León, Oaxaca, } \\
\text { Puebla, Querétaro, Quintana Roo, San Luis Potosí, } \\
\text { Sinaloa, Sonora, Tabasco, Tlaxcala, Veracruz, Yucatán y } \\
\text { Zacatecas. }\end{array}$ \\
\hline De confianza & $\begin{array}{l}\text { Aguascalientes, Baja California Norte, Baja California } \\
\text { Sur, Campeche, Chiapas, Chihuahua, Coahuila, Colima, } \\
\text { Durango, Estado de México, Guanajuato, Guerrero, } \\
\text { Hidalgo, Jalisco, Michoacán, Morelos, Nayarit, Nuevo } \\
\text { León, Oaxaca, Puebla, Querétaro, Quintana Roo, San Luis } \\
\text { Potosí, Sinaloa, Sonora, Tabasco, Tamaulipas, Tlaxcala, } \\
\text { Veracruz, Yucatán y Zacatecas. }\end{array}$ \\
\hline Supernumerarios & $\begin{array}{l}\text { Baja California Sur, Campeche, Colima, Durango, } \\
\text { Quintana Roo, Sinaloa y Tamaulipas. }\end{array}$ \\
\hline Temporales & $\begin{array}{l}\text { Aguascalientes, Guanajuato, } \quad \text { Guerrero, } \\
\text { Michoacán, Puebla y Zacatecas. }\end{array}$ \\
\hline Accidentales & Aguascalientes. \\
\hline Interinos & Chiapas, Guanajuato y Tlaxcala. \\
\hline Eventuales & $\begin{array}{l}\text { Chiapas, Chihuahua, Morelos, Querétaro y San Luis } \\
\text { Potosí. }\end{array}$ \\
\hline Extraordinarios & Chihuahua y Tamaulipas. \\
\hline Funcionarios & Chihuahua. \\
\hline Generales & Estado de México. \\
\hline
\end{tabular}




\begin{tabular}{|l|l|}
\hline \multicolumn{1}{|c|}{ Categoría } & \multicolumn{1}{c|}{ Entidades federativas } \\
\hline De base sindicalizados & Coahuila y Tamaulipas.** \\
\hline Definitivo & Jalisco. \\
\hline $\begin{array}{l}\text { De obra y tiempo } \\
\text { determinado }\end{array}$ & Tabasco. Tlaxcala. \\
\hline
\end{tabular}

* Trabajadores temporales en Jalisco también llamados supernumerarios.

** En Tamaulipas, la denominación que reciben es de "trabajadores de base sindical".

TABLA 2

ELABORACIÓN PROPIA CON DATOS DE LA LEGISLACIÓN

BUROCRÁTICA EN MATERIA DE SEGURIDAD SOCIAL

EN LAS ENTIDADES FEDERATIVAS

\begin{tabular}{|l|l|}
\hline \multicolumn{2}{|c|}{$\begin{array}{c}\text { INDICADOR 2 } \\
\text { BUCLUSIÓN-EXCEPCIÓN DE LA APLICACIÓN DE LA LEGISLACIÓN }\end{array}$} \\
\hline \multicolumn{1}{|c|}{ Entidad federativa } & \multicolumn{1}{|c|}{ Exclusión o excepción de aplicación de la normatividad } \\
\hline Aguascalientes & $\begin{array}{l}\text { Los contratados por honorarios y los que reciben } \\
\text { remuneración conjunta del Estado, Federación y municipios. }\end{array}$ \\
\hline $\begin{array}{l}\text { Baja California } \\
\text { Norte }\end{array}$ & $\begin{array}{l}\text { Los contratados bajo la legislación común o sujetos a } \\
\text { honorarios. }\end{array}$ \\
\hline Baja California Sur & No se hace exclusión o excepción. \\
\hline Campeche & $\begin{array}{l}\text { Los contratados bajo la legislación común o sujetos a } \\
\text { honorarios, trabajadores eventuales y de lista de raya. }\end{array}$ \\
\hline Chiapas & $\begin{array}{l}\text { Los contratados bajo la legislación común o sujetos a } \\
\text { honorarios. }\end{array}$ \\
\hline Chihuahua & No se hace exclusión o excepción. \\
\hline Ciudad de México & $\begin{array}{l}\text { Los que se encuentran sujetos a honorarios, por contrato } \\
\text { de obra, contrato civil, interinatos, trabajadores de la } \\
\text { Educación, }{ }^{*} \text { los que prestan servicios eventuales. }\end{array}$ \\
\hline Coahuila & No se hace exclusión o excepción. \\
\hline Colima & Los sujetos a contrato civil, mercantil o pago de honorarios. \\
\hline Durango & Los sujetos a contrato civil, mercantil o pago de honorarios. \\
\hline Estado de México & No se hace exclusión o excepción. \\
\hline Guanajuato & \\
\hline
\end{tabular}




\begin{tabular}{|c|c|}
\hline Entidad federativa & Exclusión o excepción de aplicación de la normatividad \\
\hline Guerrero & $\begin{array}{l}\text { Trabajadores al servicio de entidades paraestatales (salvo } \\
\text { convenio), policías, ministerios públicos, peritos, agentes de } \\
\text { policía ministerial, custodios y defensores de oficio. }{ }^{* *}\end{array}$ \\
\hline Hidalgo*** & No se hace exclusión o excepción. \\
\hline Jalisco & $\begin{array}{l}\text { Los que prestan servicios mediante contrato por tiempo y } \\
\text { obra determinada y por contrato civil. }\end{array}$ \\
\hline Michoacán & $\begin{array}{l}\text { Los que prestan servicios por obra o a plazo fijo, a lista de } \\
\text { raya, eventuales o emergentes y los sujetos a honorarios. }\end{array}$ \\
\hline Morelos & No se hace exclusión o excepción. \\
\hline Nayarit & $\begin{array}{l}\text { Los que prestan servicios por tiempo y obra determinada, y } \\
\text { los sujetos a la legislación común. }\end{array}$ \\
\hline Nuevo León & $\begin{array}{l}\text { Los que prestan servicios por honorarios o contrato civil, } \\
\text { eventuales con vigencia menor a seis meses } \\
\text { prestan servicios por un tiempo menor a diez horas, semana- } \\
\text { mes. }\end{array}$ \\
\hline Oaxaca & $\begin{array}{l}\text { Los que prestan servicios eventuales, los que cotizan en } \\
\text { un régimen distinto al previsto en la ley y los que perciban } \\
\text { honorarios. }\end{array}$ \\
\hline Puebla & $\begin{array}{l}\text { Los que prestan servicios por honorarios, contrato de } \\
\text { legislación común (excepto que medie convenio), contratos } \\
\text { eventuales menores a seis meses. }{ }^{* * * * *} \text { Aquellos que ingresen } \\
\text { al servicio por primera vez y hayan cumplido cincuenta años } \\
\text { sólo tienen derecho al servicio médico. }\end{array}$ \\
\hline Querétaro & No se hace exclusión o excepción. \\
\hline Quintana Roo & $\begin{array}{l}\text { Los que prestan servicios por honorario, contrato civil y } \\
\text { trabajadores eventuales. }\end{array}$ \\
\hline San Luis Potosí & No se hace exclusión o excepción. \\
\hline Sinaloa & No se hace exclusión o excepción. \\
\hline Sonora & $\begin{array}{l}\text { Los que prestan servicios por honorario, contrato civil y los } \\
\text { menores de dieciséis años. }\end{array}$ \\
\hline Tabasco & $\begin{array}{l}\text { Los prestadores de servicios profesionales, contratados por } \\
\text { la legislación civil y trabajadores eventuales (salvo convenio). }\end{array}$ \\
\hline Tamaulipas & No se hace exclusión o excepción. \\
\hline Tlaxcala & No se hace exclusión o excepción. \\
\hline
\end{tabular}




\begin{tabular}{|l|l|}
\hline \multicolumn{1}{|c|}{ Entidad federativa } & \multicolumn{1}{|c|}{ Exclusión o excepción de aplicación de la normatividad } \\
\hline Veracruz & $\begin{array}{l}\text { Los que desempeñan cargo de elección popular, } \\
\text { representantes obreros y patronales de las JCA, los que } \\
\text { laboren mediante contrato y los que reciben remuneración } \\
\text { conjunta del Estado, Federación y municipios. }\end{array}$ \\
\hline Yucatán & $\begin{array}{l}\text { Los que prestan servicios por contrato civil o laboral, } \\
\text { trabajadores a lista de raya y los que prestan servicios } \\
\text { eventuales o emergentes. }\end{array}$ \\
\hline Zacatecas & $\begin{array}{l}\text { Los que prestan servicios por honorario o asimilados } \\
\text { al salario, por contrato de prestación de servicios y los } \\
\text { trabajadores de lista de raya. }\end{array}$ \\
\hline
\end{tabular}

* Trabajadores de la educación se rigen por sus propias leyes.

** Policías, ministerios públicos, peritos, agentes de policía ministerial, custodios y defensores de oficio se rigen por sus propias leyes.

*** Hidalgo no cuenta con legislación autónoma en materia de seguridad social, se prevé un apartado especial en la ley burocrática.

**** Sólo tienen derecho al seguro de enfermedades y maternidad.

***** Sólo tienen derecho a servicio médico mediante convenio. 